\title{
Low molecular weight heparin as an adjunct to thrombolysis for acute myocardial infarction: the FATIMA study
}

\author{
S A J Chamuleau, R J de Winter, M Levi, R Adams, H R Büller, M H Prins, K I Lie, \\ R J G Peters, on behalf of the Fraxiparin Anticoagulant Therapy in Myocardial Infarction \\ Study Amsterdam (FATIMA) Study Group
}

Department of Cardiology, Academic Medical Centre, University of Amsterdam, Netherlands S A J Chamuleau $\mathrm{R} \mathrm{J}$ de Winter $\mathrm{R}$ Adams K I Lie R J G Peters

Centre for Haemostasis, Thrombosis, Atherosclerosis and Inflammation Research, Academic Medical Centre, University of Amsterdam

M Levi H R Büller

Department of Clinical Epidemiology and Biostatistics, Academic Medical Centre, University of Amsterdam M H Prins

Correspondence to: Dr R J de Winter, Academic Medical Centre, Department of Cardiology, room G3-231, Meibergdreef 9, $1105 \mathrm{AZ}$ Amsterdam, Netherlands email: r.j.dewinter@ amc.uva.nl

Accepted for publication 16 February 1998

\begin{abstract}
Objective-To investigate the feasibility of fixed dose, weight adjusted subcutaneous low molecular weight heparin (LMWH), with monitoring of anti-Xa levels and assessment of coronary patency rates after three to five days, thereby giving an initial indication of its safety and efficacy. Design-In 30 patients with acute myocardial infarction, LMWH (nadroparine) was given as a body weight adjusted intravenous bolus with thrombolysis (rt-PA infusion) and in weight adjusted subcutaneous doses at six hours, and every 12 hours thereafter for 72 hours. The target range was defined prospectively as 0.35 0.70 anti-factor Xa activity (aXa) units. The aXa level was measured every six hours. Coronary angiography was performed in all patients within five days after the start of thrombolytic treatment to determine patency (TIMI 2 and 3 flow) of the infarct related artery.

Results-The mean (SEM) aXa level over 72 hours was $0.52(0.08) \mathrm{U} / \mathrm{ml}$; from 12 hours onwards $88 \%$ of all aXa measurements were within the target range. At angiography, a patent infarct related artery was present in 24 of the 30 patients. No major bleeding complications occurred, though minor bleeding complications were observed in two patients.

Conclusions-This small study indicates that LMWH is feasible as an adjunct to thrombolysis in patients with acute myocardial infarction. The aXa levels were within the target range and patency rates at three to five days were around $80 \%$, with no major bleeding complications.

(Heart 1998;80:35-39)
\end{abstract}

Keywords: acute myocardial infarction; thrombolysis; low molecular weight heparin; FATIMA study

It is well established that the use of thrombolytic treatment in patients with acute myocardial infarction results in early coronary artery patency, salvation of left ventricular function, and improved survival. ${ }^{12}$ The second international study of infarct survival (ISIS-2) showed conclusively the substantial value of combined aspirin and thrombolytic treatment (streptokinase) in patients with suspected acute myocardial infarction, and GUSTO-I showed a better efficacy of accelerated tissue plasminogen activator (rt-PA) compared with streptokinase. ${ }^{34}$ Although heparin is widely used as an adjunct to rt-PA treatment in patients with acute myocardial infarction, large clinical trials suggest only a potentially beneficial effect. ${ }^{5-8}$ Trials with angiographic end points indicate that the adjunctive use of intravenous unfractionated heparin improves the outcome; however, this has not been unequivocally shown in large randomised trials with clinical outcomes. ${ }^{9-13}$ Evidence from subsets of patients in the SCATI and GISSI-2 trials suggests that the administration of high dose subcutaneous heparin results in better survival than when no heparin is given (mortality $4.5 \%$ among patients receiving heparin $v 8.8 \%$ among patients who did not receive heparin). ${ }^{56}$ Although the results of these non-randomised comparisons have to be interpreted with caution, most cardiologists have adopted the use of heparin as an adjunct to rt-PA thrombolysis in patients with acute myocardial infarction. The addition of unfractionated heparin to thrombolytic treatment resulted in a slightly higher incidence of bleeding, but no differences in the incidence of haemorrhagic strokes were reported. ${ }^{14}$

Because of the unpredictable bioavailability of unfractionated heparin, frequent monitoring of the anticoagulant effect is necessary, with multiple dose adjustments. ${ }^{15}$ Even so, many patients are not in the target therapeutic range for a substantial period of time. For example, in the GUSTO-I study nearly $50 \%$ of the heparin treated patients had an activated partial thromboplastin time (aPTT) value below the target range of 60-85 seconds at 24 hours. ${ }^{4}$

In recent years, low molecular weight heparins (LMWH) have been developed. Compared with unfractionated heparin, these compounds have a longer plasma half life, less variability in their anticoagulant response to fixed doses, and possibly a more favourable ratio of antithrombotic to haemorrhagic effects. ${ }^{16}{ }^{17}$ These favourable properties of LMWH have led to the concept that the subcutaneous administration of LMWH produces therapeutic anticoagulant levels without the need for laboratory monitoring. Instead, the dose of LMWH is based on the body weight of the patient and remains fixed for the duration of the treatment. Various studies in patients with venous thromboembolism have shown that treatment with fixed dose subcutaneous LMWH is at least as effective and safe as treatment with dose adjusted intravenous 
unfractionated heparin. ${ }^{18} 19$ LMWH has also been studied in unstable angina patients, ${ }^{20-22}$ and in one dose finding study following thrombolytic treatment. ${ }^{23}$

There is little information about the use of LMWH in conjunction with thrombolytics in the setting of acute myocardial infarction, in particular with respect to bleeding complications. We have therefore performed a study to investigate the feasibility of giving fixed dose, weight adjusted subcutaneous LMWH in patients receiving thrombolytic treatment for acute myocardial infarction, with monitoring of anti-Xa levels, thus establishing an initial indication of its safety and efficacy.

\section{Methods}

DESIGN

This study was designed as a small (30 patients) open study to investigate feasibility and to obtain a first indication of the efficacy and safety of the proposed treatment schedule. The protocol was approved by the institutional ethics review board.

\section{PATIENTS}

Consecutive patients with acute myocardial infarction who were eligible to receive thrombolytic treatment were included in the study, according to the GUSTO-I criteria ${ }^{24}$ : presentation within six hours of onset of chest pain suggestive of myocardial ischaemia; ST segment elevation of at least $0.2 \mathrm{mV}$ in two or more contiguous precordial electrocardiographic leads or at least $0.1 \mathrm{mV}$ in two or more contiguous standard electrocardiographic leads; and exclusion of patients with haemorrhagic diathesis, known haematological abnormalities, active bleeding, severe uncontrolled hypertension, cerebrovascular disease, recent cranial trauma, recent surgery or prolonged resuscitation, and serious medical illnesses. Further exclusion criteria were: age less than 18 years, use of oral anticoagulants or intravenous heparin, and pregnancy. All patients gave written informed consent.

\section{TREATMENT}

Eligible patients received recombinant tissuetype plasminogen activator (rt-PA) (Actilyse; Boehringer Ingelheim, Germany) to a maximum of $100 \mathrm{mg}$ according to the accelerated dosing scheme as used in the GUSTO-I trial. ${ }^{4}$ Adjunctive treatment with subcutaneous LMWH (nadroparine) (Fraxiparin; Sanofi, France) was initiated with an intravenous bolus of $100 \mathrm{U} / \mathrm{kg}$ just before the start of the rt-PA infusion, followed by subcutaneous injections at six hours and every 12 hours for 72 hours. The dose of the subcutaneous injections was stratified according to the body weight of the patient (<50 kg: 10000 anti-factor $\mathrm{Xa}(\mathrm{aXa})$ U; 50-70 kg: 15000 aXa U; > 70 kg: 20000 aXa U). This dose was based on clinical trials of subcutaneous LMWH (nadroparine) in which efficacy was proved in patients with venous thromboembolism, with few bleeding complications. ${ }^{18}{ }^{25}$ All patients received $300 \mathrm{mg}$ carbasalate calcium (Ascal; Dagta Pharma,
Diemen, The Netherlands) on admission to the coronary care unit and subsequently 100 mg daily.

\section{MONITORING}

Initial successful reperfusion was assessed noninvasively, according to previously described criteria. ${ }^{26}{ }^{27}$ Indicators of early reperfusion were recorded every hour during the first six hours after rt-PA infusion. The reperfusion indices consisted of electrocardiographic (ECG) changes ( $>50 \%$ decrease in ST elevation), the presence of reperfusion arrhythmias, improvement in clinical signs, and a peak creatine kinase $(\mathrm{CK}-\mathrm{MB})$ at or before 12 hours after the onset of symptoms.

All patients were closely monitored throughout the study period for the occurrence of signs of reocclusion or bleeding complications. Monitoring included frequent symptom inquiry, physical examination, ECG, laboratory investigations, and coronary angiography at the end of the study period.

Laboratory measurements included assessment of the anti-factor Xa activity (aXa) every six hours. Blood was collected in plastic syringes containing $3.2 \%$ sodium citrate. Platelet-poor plasma was obtained by centrifugation for 20 minutes at $2000 \times g$ and at $4^{\circ} \mathrm{C}$. The chromogenic anti-factor $\mathrm{Xa}$ activity assay was performed using an EPOS 5060 analyser, as described previously, and calibrated against a nadroparine standard. ${ }^{28} \mathrm{An}$ anti-Xa activity of $0.35-0.70 \mathrm{U} / \mathrm{ml}$ was defined as the target range. Previous studies have found that there is a favourable in vivo anticoagulant activity in this aXa range. ${ }^{29}$ Additionally, CK-MB was assessed every six hours, and haemoglobin once a day.

\section{CORONARY ANGIOGRAPHY}

Angiography was performed according to the study protocol three to five days after the start of thrombolytic treatment, unless earlier examination was indicated. Patients with recurrent typical chest pain and ECG abnormalities consistent with myocardial ischaemia refractory to medical treatment within the study period were eligible for earlier coronary angiography. The end point in these patients was considered to be after angiography was performed and further treatment was at the discretion of the attending cardiologist. Coronary angiography was performed according to standard procedures and recorded on cinefilm. At least one pair of orthogonal projections of the infarct related coronary artery (IRA) and of the uninvolved artery were acquired. Patency was assessed on first contrast injection after intracoronary injection of glyceryl trinitrate. Coronary flow was expressed as TIMI flow, and vessels with TIMI 2 and 3 flow were considered "patent"; vessels with TIMI 0 and 1 flow were considered "occluded." 31 Two independent observers from other institutions, who were unaware of patient data and study questions, determined the IRA on the basis of the admission ECGs and scored the TIMI flow. In case of disagreement concerning 
the IRA or the TIMI flow, the observations of a third independent observer were decisive.

OUTCOMES

The feasibility outcome was defined as the ability to give LMWH twice daily subcutaneously in patients who have received thrombolytic treatment for acute myocardial infarction, and the ability of the proposed dosing scheme of LMWH to provide constant and adequate levels of anticoagulation, as assessed by antifactor Xa levels.

An outcome indicating possible efficacy was defined as angiographically proven patency (TIMI grade 2 and 3 flow) of the infarct related reperfused vessel.

An outcome indicating possible safety was defined by the incidence of clinically apparent major or minor haemorrhage during the first three days of hospital admission. Bleeding complications were divided in major (clinically overt associated with a fall in haemoglobin concentration of more than $2 \mathrm{mmol} / 1$ or requiring a transfusion of at least one unit of packed red cells, or when the haemorrhage was intracranial) and minor (clinical bleeding, which did not meet the criteria for major bleeding).

STATISTICAL AND SAMPLE SIZE CONSIDERATIONS The GUSTO angiographic substudy indicated that the patency rate after rt-PA and intravenous unfractionated heparin (after five to seven days) is approximately $84 \% .^{32}$ In our small pilot study we prospectively expected patency rate to be achieved in at least 22 of 30 patients $(73 \% ; 95 \%$ confidence interval $54.1 \%$ to $87.7 \%$ ), to justify the initiation of a larger randomised trial. Confidence intervals were calculated with the binomial distribution for proportions.

Results

Baseline characteristics are given in table 1.

CLINICAL OUTCOMES AND ANGIOGRAPHIC

PATENCY

All patients underwent angiography within five days (median 3, range 0.5 to 5). Patency of the IRA was present in 24 of the 30 patients (patency rate of $80 \%, 95 \%$ confidence interval $61.4 \%$ to $92.3 \%$ ). The two independent observers disagreed in one patient on the IRA. Disagreement over patency occurred in one other case. IRA and TIMI flow results are shown in table 2 .

In 10 patients, coronary angiography was performed within three days, which was earlier than required by the study protocol. In three patients this was because of rescue angioplasty, one had with failed thrombolysis (TIMI flow 0 ), one had threatening acute reclosure after initial reperfusion (TIMI flow 3), and one had persistent ST elevation (TIMI flow 3). Angiography was performed in seven patients because of recurrent symptoms within three days (four patent IRAs, three IRAs with TIMI flow 0 or 1). Angioplasty (PTCA) was performed in nine of the 10 patients who underwent angiography within three days. The
Table 1 Baseline characteristics on admission

\begin{tabular}{lll}
\hline Number of patients & & 30 (male 22) \\
& Age (years) & $61(30-81)$ \\
& Weight (kg) & $75(45-120)$ \\
& Heart rate (beats/min) & $70(41-123)$ \\
& Systolic BP (mm Hg) & $123(90-180)$ \\
Risk factors & Smoking & $18(60 \%)$ \\
& Diabetes & $4(13.3 \%)$ \\
& Hypertension & $10(33.3 \%)$ \\
& Hypercholesterolaemia & $4(13.3 \%)$ \\
Cardiac history & Family history of CAD & $12(40 \%)$ \\
& Previous AMI & 2 \\
& Previous PTCA & 1 \\
Infarction & Previous CABG & 0 \\
& Interval (hours) & $2.9(0.5-6)$ \\
& Anterior & 10 \\
& Inferior & 20 \\
Nadroparin regimen & Peak CK-MB (U/1) & $139(5-786)$ \\
& 10000 aXa Units/12 h & 1 \\
& 15000 aXa Units/12 h & 7 \\
& 20000 aXa Units/12 h 22 & 22
\end{tabular}

Values are median (range). The interval from onset of symptom to admission is shown. Nadroparin treatment started with a bolus of $100 \mathrm{aXa} \mathrm{U} / \mathrm{kg}$. The nadroparin regimen (from 6 hours onwards every 12 hours) was stratified according to the patient's body weight: < $50 \mathrm{~kg}, 10000$ aXa U; 50-70 kg, 15000 aXa U; and $>70 \mathrm{~kg}, 20000 \mathrm{aXa} U$ per subcutaneous injection. AMI, acute myocardial infarction; aXa, anti-factor Xa activity; $\mathrm{BP}$, blood pressure; CABG, coronary artery bypass grafting; CAD, coronary artery disease; PTCA, percutaneous transluminal coronary angioplasty.

Table 2 Clinical outcomes

\begin{tabular}{cll}
\hline $\begin{array}{l}\text { Signs of early reperfusion } \\
\text { Coronary angiography } \\
\text { (number of patent }\end{array}$ & Rescue angioplasty & 25 \\
infarct related arteries) & Recurrent angina & $7(4)$ \\
& Elective & $20(18)$ \\
Infarct related artery & RCA & 16 \\
& LAD & 9 \\
TIMI flow & LCx & 5 \\
& 0 & 5 \\
& I & 1 \\
Bleeding complications & II & 2 \\
& III & 22 \\
& Major & 0 \\
& Minor & 2
\end{tabular}

LAD, left anterior descending coronary artery; LCx, left circumflex coronary artery; RCA, right coronary artery.

other 20 patients underwent elective coronary angiography between three and five days, according to the study protocol (18 patent IRAs, two occluded IRAs).

In five patients no signs of early reperfusion were seen after 90 minutes. Rescue PTCA was performed in three of these patients (see above). The other two patients did not undergo rescue PTCA, one because of electrocardiographically small infarct size, and one infarction complicated by an episode of ventricular fibrillation accompanied by equivocal ST segment changes after defibrillation.

No major bleeding complications occurred, and there were two minor bleeding complications (one venepuncture haematoma and one urinary tract bleeding).

ANTI-FACTOR Xa

The mean aXa measurements of 30 patients for each time point are presented in fig 1 . At six hours after admission, the mean aXa level was $0.28 \mathrm{U} / \mathrm{ml}$. At that time, five of the 30 measurements were within the target range. From 12 hours onwards $88 \%$ of the aXa measurements were within the target range. No differences in aXa levels were observed between the three dosing strategies (data not shown) and there were no differences between the 


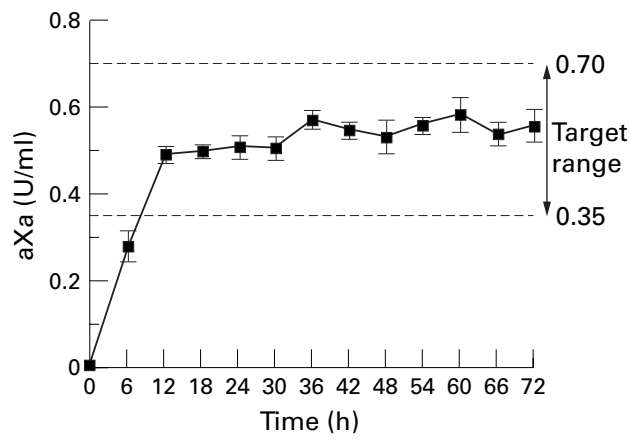

Figure 1 The plasma anti-factor Xa activity (aXa; $\mathrm{U} / \mathrm{ml}$ ) measured at 13 time points: just before the start of thrombolytic treatment, six hours after the start, at 12 hours, and every six hours thereafter up to 72 hours after the start of rt-PA infusion. The mean values of the 30 patients per time point are depicted, with their SEM values. The predefined target range was 0.35-0.70 U/ml. From 12 hours onwards $88 \%$ of all values were within this range.

patients $(n=20)$ who underwent angiography before day 3 because of symptoms compared with the patients $(n=10)$ who underwent angiography between days 3 and 5 (mean (SEM) values of $0.52(0.04)$ and $0.50(0.07)$, respectively; NS). The mean aXa level over 72 hours was $0.52(0.08) \mathrm{U} / \mathrm{ml}$.

\section{Discussion}

This study shows that the use of fixed dose, weight adjusted subcutaneous LMWH as an adjunct to rt-PA thrombolytic treatment for acute myocardial infarction induces stable and predictable aXa levels within the predefined target range. Patency rates at three to five days $(80 \%)$ were comparable with the rt-PA arm $(84 \%)$ in the GUSTO angiographic substudy. ${ }^{32}$ No major bleeding complications occurred. Hence this study shows that treatment with LMWH (nadroparine) is feasible and probably effective and safe as an adjunct to thrombolysis in patients with acute myocardial infarction. In clinical practice, treatment with LMWH does not require labourious laboratory monitoring and dose adjustments, which is a major advantage. Therefore, even if treatment with LMWH were no more effective than with unfractionated heparin, it would still have an important practical advantage. In addition, stable and predictable levels of anticoagulation are expected to result in fewer bleeding complications. As shown in the GUSTO-I study, aPTT levels above 85 seconds are associated with excess bleeding. ${ }^{24}$

The effective therapeutic range for $\mathrm{LMWH}$ as adjunctive treatment is unknown. One dose finding study of LMWH treatment following thrombolysis in acute myocardial infarction presumed a target range of $0.6-1.0$ anti-Xa $\mathrm{U} / \mathrm{ml}$ (based on treatment with deep venous thrombosis). ${ }^{23}$ In that study, daltaparin was used after thrombolysis with streptokinase; no major bleeding complications occurred; however, eight urogenital tract bleeding episodes and 17 venepuncture haematomas were described. In our study we used nadroparine and rt-PA, and just two minor bleeding complications occurred (presumed target range 0.350.70 anti-Xa U/ml).
We considered TIMI flow 2 and 3 as patent, as have other patency studies. ${ }^{9}{ }^{10} 1231$ However, the GUSTO angiographic substudy indicated that among patients with partial flow in the IRA (TIMI flow 2), ventricular function was worse and mortality higher than among patients with normal flow (TIMI flow 3). ${ }^{32}$ In our study TIMI flow 3 was present in 22 of 30 IRAs (73\%); in the GUSTO trial $58 \%$ of the rt-PA arm showed TIMI 3 flow.

At time point six hours after start of thrombolysis, 25 of the 30 aXa levels were below the predefined target range, indicating that the dose of the initial bolus injection may have been too low. However, from 12 hours onwards the aXa levels showed means and SEM values within the target range for all time points.

As a possible alternative to treatment with unfractionated heparin, studies have been conducted with direct thrombin antagonists. However, studies using conjunctive treatment with hirudin have been disappointing. For instance, the HIT-III trial, comparing hirudin with heparin, showed the therapeutic range of the former to be narrower than with unfractionated heparin; this was consistent with the findings of the GUSTO-II and the TIMI-9 trials. ${ }^{33-35}$

Our study was uncontrolled, comprising a small patient group, and was not powered for proper assessment of clinical outcomes. Furthermore, there was no angiographic "proof" of early successful reperfusion with an angiogram at six hours. However, the study was designed as an initial pilot study to establish the feasibility of giving LMWH as an adjunct to thrombolysis in patients with acute myocardial infarction, and to obtain information about patency rates and bleeding complications. More definitive proof of the efficacy and safety of LMWH must come from a larger randomised trial comparing unfractionated heparin with LMWH as adjuncts to thrombolytic treatment. Such studies are in preparation.

We thank A E R Arnold, MD (Medical Centre Alkmaar, The Netherlands) and G Hoedemaker, MD (Gooi-Noord Hospital, Blaricum, The Netherlands) for independently determining IRA and TIMI flow rates. The skilful support of the nurses of the coronary care unit of the Academic Medical Centre in Amsterdam is gratefully acknowledged. This study was sponsored by Sanofi, France.

1 Fuster V. Coronary thrombolysis - a perspective for the practicing physician. $N$ Engl F Med 1993;329:723-5.

2 Fibrinolytic Therapy Trialists' (FFT) collaborative group. Indications for fibrinolytic therapy in suspected myocardial infarction: collaborative overview of early mortality and major morbidity results from all randomised trials of more major morbidity results from all randomised trial

3 ISIS-2 (second international study of infarct survival) Collaborative Group. Randomised trial of intravenous streptokinase, oral aspirin, both, or neither among 17,187 cases of suspected myocardial infarction: ISIS-2. Lancet 1988;ii: 349-60.

4 Granger CB, Hirsh J, Califf RM, et al. Activated partial thromboplastin time and outcome after thrombolytic therapy for acute myocardial infarction. Results from the GUSTO-I trial. Circulation 1996;93:870-8.

5 Gruppo Italiano per lo studio della sopravvivenza nell' infarto miocardico. GISSI-2: a factorial randomised trial of alteplase versus streptokinase and heparin versus no heparin among 12,490 patients with acute myocardial infarction. Lancet 1990;336:65-71.

6 The SCATI (Studio sulla calciparina nell'angina e nella trombosi ventricolare nell'infarcto) Group. Randomised controlled trial of subcutaneous calcium-heparin in acute myocardial infarction. Lancet 1989;ii: 182-6.

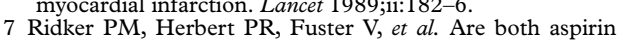
and heparin justified as adjuncts to thrombolytic therapy for acute myocardial infarction? Lancet 1993;341:1574-7. 
8 Wood AJJ. Aspirin, heparin, and fibrinolytic therapy in suspected acute myocardial infarction. $N$ Engl $\mathcal{f} \mathrm{Med}$ pected acute my

9 Bleich SD, Nichols TC, Schumacher RR, et al. Effect of heparin on coronary arterial patency after thrombolysis with tissue plasminogen activator in acute myocardia infarction. Am f Cardiol 1990;66:1412-17.

10 Arnout J, Simoons ML, de Bono D, et al. Correlation between level of heparinization and patency of the infarctrelated coronary artery after treatment of acute myocardia infarction with alteplase (rt-PA). $₹ \mathrm{Am}$ Coll Cardiol 1992;20:513-19.

11 De Bono DP, Simoons ML, Thijssen J, et al. Effect of early intravenous heparin on coronary patency, infarct size, and bleeding complications after alteplase thrombolysis: results of a randomised double blind European cooperative study group trial. Br Heart 7 1992;67:122-8.

12 Hsia J, Hamilton WP, Kleiman N, et al, for the HART Investigators. A comparison between heparin and low-dose aspirin as adjunctive therapy with tissue plasminogen actiaspirin as adjunctive therapy with tissue plasminogen acti-
vator for acute myocardial infarction. $N$ Engl $\mathcal{F ~ M e d}$ vator for acute myo

13 Prins MH, Hirsh J. Heparin as an adjunctive treatment after thrombolytic therapy for acute myocardial infarction. $A m \mathcal{F}$ Cardiol 1991;67:3-11A.

14 ISIS-3 (Third international study of infarct survival) Collaborative Group. ISIS-3: a randomised comparison of streptokinase versus tissue plasminogen activator versus anistreplase and of aspirin plus heparin versus aspirin alone among 41,299 cases of suspected acute myocardial infarction. Lancet 1992;339:753-69.

15 Kroon C, ten Hove WR, de Boer A, et al. Highly variable anticoagulant response after subcutaneous administration of high-dose (12,500 IU) heparin in patients with myocardial infarction and healthy volunteers. Circulation 1992:86: 1370-5.

16 Emanuele RM, Fareed J. The effect of molecular weight on the bioavailability of heparin. Thromb Res 1987;48:591-6.

17 Hirsh J, Fuster V. Guide to anticoagulant therapy. Part 1: heparin. Circulation 1994;89:1449-68.

18 Koopman MM, Prandoni P, Piovella F, et al. Treatment of venous thrombosis with intravenous unfractionated heparin administered in the hospital as compared with subcutaneous low-molecular-weight heparin administered at home. The TASMAN Study Group. N Engl F Med 1996; 334:682-7.

19 Ten Cate JW, Koopman MM, Prins MH, et al. Treatment of venous thromboembolism. Thromb Haemost 1995;74:197203.

20 FRISC (Fragmin during instability in coronary artery disease) Study Group. Low-molecular-weight heparin during instability in coronary artery disease. Lancet 1996;347: 561-8.

21 Klein W, Buchwald A, Hillis SE, et al. Comparison of low-molecular-weight heparin with unfractionated heparin low-molecular-weight heparin with unfractionated heparin 政 unstable coronary artery disease (Fragmin in unstable coronary
22 The Thrombolysis in Myocardial Infarction (TIMI) IIA Trial Investigators. Dose-ranging trial of enoxaparin for unstable angina: results of TIMI IIA. $\mathcal{f} \mathrm{Am}$ Coll Cardiol 1997;29:1474-82.

23 Strandberg LE, Kahan T, Lundin P, et al. Anticoagulant effects of low-molecular-weight heparin following thrombolytic therapy in acute myocardial infarction: a dosefinding study. Haemostasis 1996;26:247-57.

24 The GUSTO-investigators. An international randomized trial comparing four thrombolytic strategies for acute myocardial infarction. N Engl f Med 1993;329:673-82.

25 Prandoni P, Lensing AW, Büller HR, et al. Comparison of subcutaneous low-molecular-weight heparin with intravenous standard heparin in proximal deep-vein thrombosis. Lancet 1992;339:441-5.

26 Klootwijk P, Cobbaert C, Fioretti P, et al. Noninvasive assessment of reperfusion and reocclusion after thrombolyis in acute myocardial infarction. Am $\mathcal{7}$ Cardiol 1993;72: $75-84 \mathrm{G}$.

27 Hohnloser SH, Zabel M, Kasper W, et al. Assessment of coronary artery patency after thrombolytic therapy: accurate prediction utilizing the combined analysis of three noninvasive markers. F Am Coll Cardiol 1991;18:44-9.

28 Ten Cate H, Lamping RJ, Henny ChP, et al. Automated amidolytic method for determining heparin, a heparinoid and a low molecular weight heparin fragment based on and a low molecular weight heparin fragment

29 Tew CJ, Lane DA, Thompson E, et al. Relationship between ex vivo anti-proteinase (factor $\mathrm{Xa}$ and thrombin) assays and in vivo anticoagulant effect of very low molecular weight heparin, CY222. Br f Haematol 1988;70:335-40.

30 TIMI Study Group. The thrombolysis in myocardial infarction (TIMI) trial: phase I findings. N Engl f Med 1985;312: 932-6.

31 Chesebro JH, Knatterud G, Roberts R, et al. Thrombolysis in myocardial infarction (TIMI) trial, phase 1: a comparison between intravenous tissue plasminogen activator and intravenous streptokinase: clinical findings through hospital discharge. Circulation 1987;76:142-54.

32 The GUSTO Angiographic Investigators. The effects of tissue plasminogen activator, streptokinase, or both on coronary-artery patency, ventricular function, and survival after acute myocardial infarction. $N$ Engl F Med 1993;329: 1615-22.

33 Neuhaus KL, van Essen R, Tebbe U, et al. Safety observations from the pilot phase of the randomised r-hirudin for improvement of thrombolysis (HIT-III) study. Circulation 1994;90:1638-42.

34 Antman EM, for the TIMI 9A investigators. Hirudin in acute myocardial infarction. Safety report from the thrombolysis and thrombin inhibition in myocardial infarction (TIMI) 9A trial. Circulation 1994;90:1624-30.

35 The global use of strategies to open occluded coronary arteries (GUSTO) IIa investigators. Randomized trial of intravenous heparin versus recombinant hirudin for acute coronary syndromes. Circulation 1994;90:1631-7. 